\title{
VALORACIÓN DEL PROCESO FORMATIVO DE LOS MONITORES DE UN PROGRAMA DE DEPORTE ESCOLAR EDUCATIVO
}

Assessment of the training process of the instructors of an after-school sports programme

Avaliação do processo formativo de monitores de um programa de desporto escolar educativo

Félix Enrique Lobo de Diego (1)

Juan Carlos Manrique Arribas (2)

Agradecemos al Ministerio de Educación, Cultura y Deporte de España la financiación del proyecto de investigación en el que se engloba este estudio, a través de una Ayuda para la Formación de Profesorado Universitario (FPU).

(1) Universidad de Valladolid, España. Teléfono: +34 921112241. Correo electrónico: felixenrique.lobo@uva.es

(2) Universidad de Valladolid, España. Teléfono: +34 921112241. Correo electrónico: juancarlos.manrique@uva.es

\section{Resumen}

En esta investigación se estudian las valoraciones del proceso formativo de los monitores del Programa Integral de Deporte Escolar de Segovia en el que está implícito la evaluación formativa. Para ello se ha utilizado una metodología de corte cualitativo, en la que se emplea la técnica de análisis documental para obtener la información. Nuestras fuentes documentales han sido 4 memorias de monitores deportivos, que estos entregan al finalizar el curso. Como resultado principal se observa que la evaluación continua que se realiza en el proceso formativo de los monitores sirve para mejorar el proceso de enseñanza y aprendizaje. La investigación permite concluir que los monitores están satisfechos con la formación recibida y que el proceso continuo de evaluación permite que mejoren sus cualidades docentes como monitores deportivos, lo que repercute en la mejora del proceso de enseñanza y aprendizaje.

Palabras clave: Deporte escolar; evaluación formativa; monitores; memorias

\section{Abstract}

In this research we study the training process of the sport monitors of the Segovia AfterSchool Sports Program in which the formative assessment is implicit. For this purpose, 
a qualitative methodology was used and the documentary analysis technique was used to obtain the information. Our documentary sources have been 4 memories of sports monitors. As a main result it is observed that the continuous evaluation that is carried out in the training process of the monitors serves to improve the teaching and learning process. The research allows us to conclude that the monitors are satisfied with the training received, and that the continuous assessment process allows to improve the teaching qualities of the sports monitors, which has an impact on the improvement of the teaching and learning process.

Keywords: After-School sport; formative assessment; sport monitors; documentary memories

\section{Resumo}

Nesta investigação estudam-se as avaliações do processo formativo de monitores do Programa Integral de Desporto Escolar de Segóvia, no qual está implícito a avaliação formativa. Para o efeito, utilizou-se uma metodologia qualitativa, através da técnica de análise documental para obter as informações. Como fontes documentais foram usadas quatro memórias dos monitores desportivos, entregues no final do curso. Como resultado principal observa-se que a avaliação contínua realizada no processo formativo dos monitores serve para melhorar o processo de ensino e aprendizagem. A investigação permite concluir que os monitores estão satisfeitos com a formação recebida e que o processo contínuo de avaliação permite-lhes melhorar as suas qualidades docentes como monitores desportivos, o que se repercute na melhoria do processo de ensino e aprendizagem.

Palavras-chave: Desporto escolar; avaliação formativa; monitores; memórias

\section{Introducción}

En el Programa de Deporte Escolar del Municipio de Segovia (PIDEMSG), un programa con carácter polideportivo y basado en el modelo comprensivo de iniciación deportiva, desarrollado durante los años 2009 y 2018, las personas que participaban como monitores deportivos, tanto becados (estudiantes de los Grados de Educación de la Facultad de Educación de Segovia) como contratados (maestros titulados), recibían una formación permanente y continua que se iba adaptando cada año en función de las 
circunstancias y necesidades detectadas en las sucesivas evaluaciones anuales (PérezBrunicardi, Álvaro-Garzón \& López-Pastor, 2018).

A lo largo de todo este proceso está implícita la evaluación formativa, puesto que este Programa está en continua revisión durante todo el curso académico a través de los ciclos de investigación-acción que realiza el equipo investigador con la ayuda de los coordinadores y los monitores del PIDEMSG. También mediante la continua supervisión y feedback que reciben los monitores del Programa por parte de los coordinadores tras la implementación de las sesiones de aquellos, o a través de las reuniones de seguimiento que se realizan entre el equipo investigador y monitores para realizar ajustes en la programación o en los cursos monográficos que se organizan. Todo ello con el objetivo de mejorar la calidad del aprendizaje de los jóvenes deportistas y el de ayudar a los monitores en el desempeño de su práctica docente. En todo momento se pretende conseguir los fines que cualquier evaluación debe cumplir, como así lo expresa López-Pastor (2006): mejorar los procesos de enseñanza-aprendizaje, aprender más y perfeccionar la práctica docente.

Dicho proceso de formación está compuesto por un curso de formación inicial, seminarios semanales o quincenales a modo de asamblea, y una memoria de prácticas (Martínez-Scott, López-Pastor \& Pérez-Brunicardi, 2012; Pérez-Brunicardi, ÁlvaroGarzón \& López-Pastor, 2018). En este estudio nos vamos a centrar en la memoria que tienen que realizar durante el curso escolar. Esta memoria es un documento que estos profesionales van completando con la programación de las actividades y las valoraciones y reflexiones de su implementación, con la intención de favorecerse de los beneficios que ofrece la evaluación formativa y continua para el aumento de la calidad del proceso de enseñanza-aprendizaje. Esta memoria es supervisada cada tres meses por los coordinadores, con la finalidad de mejorar no solamente en sus cualidades como docentes sino en mejorar la calidad del proceso de enseñanza-aprendizaje; estando por consiguiente centradas en la mejora de los aprendizajes de los monitores y escolares.

\section{Método}

Nuestra investigación es de corte cualitativo, en la que hemos utilizado el análisis documental como técnica de recogida de información. Nuestra fuente documental son 8 memorias, de las cuales 3 pertenecen a monitores becados y 1 a monitores contratados en el curso 2016-2017 en el PIDEMSG, como ejemplos de las que se han realizado a lo 
largo de todos los años de implementación del Programa. En ellas se ha puesto el foco en las valoraciones finales que los investigados realizan de todo el proceso formativo recibido y los temas que son mencionados en ellas. Para facilitar la organización de la información recogida hemos agrupado los datos en torno a los siguientes grupos:

Tabla 1.

Categorías de análisis cualitativo

\begin{tabular}{ll}
\hline & \multicolumn{1}{c}{ Categoría } \\
\hline 1 & Satisfacción con el proceso formativo \\
2 & Aprendizajes de monitores y escolares \\
3 & Empleo de metodologías alternativas y participativas en iniciación deportiva \\
4 & Utilidad y transferencia a otros contextos \\
\hline
\end{tabular}

Cada una de las memorias ha sido codificada siguiendo el procedimiento que aparece en la Tabla 2.

Tabla 2.

Sistema de codificación empleado en las memorias

\begin{tabular}{|c|c|c|c|}
\hline Memoria & Número & Participantes & Tipo de monitor \\
\hline $\mathrm{ME}$ & De 01 a 04 & MO: Monitor & $\begin{array}{l}\text { BE: Becado } \\
\text { CO: Contratado }\end{array}$ \\
\hline
\end{tabular}

\section{Resultados}

A continuación, mostramos de forma breve los principales resultados de este estudio. En primer lugar, los monitores señalan estar satisfechos con el proceso formativo y continuo que se lleva a cabo en el PIDEMSG, como se observa en la Figura 1, pero los monitores manifiestan que para que este proceso sea más enriquecedor sería adecuada una mayor regularidad en la asistencia a los seminarios por parte de todos los monitores (Figura 2).

Para finalizar, cabe destacar que estoy satisfecho en que haya un programa de formación hacia una educación globalizada en la que hay coordinadores que te proporcionan ayuda, ofreciendo una educación de la mayor calidad posible aplicando metodologías que se consideran novedosas e innovadoras. (ME01MOBE, p. 77)

Figura 1.

Extracto de una memoria sobre la satisfacción con el proceso formativo recibido 
En cuanto al curso de formación del PIDEMSG, cabe mencionar mi descontento, ya que

a los seminarios siempre hemos asistido tres personas. Esto limita el intercambio de opiniones y situaciones vividas en los centros, lo cual nos enriquecería si todos asistiesen a los seminarios de formación. (ME01MOBE, p. 76)

Figura 2.

Fragmento de una memoria sobre aspectos a mejorar en la formación recibida

Siguiendo con los aprendizajes, hallamos que tanto los monitores como los escolares del PIDEMSG aprenden a lo largo del curso nociones deportivas, poniendo especial énfasis en favorecer una educación en valores y actitudes positivos que permitan las relaciones sociales entre los escolares y los monitores (Figura 3). Además, encontramos que el empleo de las metodologías alternativas y participativas en iniciación deportiva favorecen los aprendizajes significativos de los escolares como se aprecia en la Figura 4.

Los alumnos de Educación Infantil han aprendido a seguir unas normas, aunque a los más pequeños les ha costado algo más de tiempo y esfuerzo. En cuanto al aprendizaje deportivo los niños han aprendido nuevos juegos que les han ayudado a mejorar sus capacidades físicas y sociales. (ME04MOCO, p. 7)

Figura 3.

Parte de una memoria en la que se versa sobre los aprendizajes de los escolares

Finalmente, el objetivo que consiguieran unos aprendizajes más significativos creo que sí que lo he conseguido, debido a la metodología empleada. He usado paradas de reflexión-acción para comprobar si han aprendido bien los contenidos trabajados, al igual que las diferentes asambleas. (ME03MOBE, p. 90)

Figura 4.

Extracto de una memoria en la que se comenta sobre los aprendizajes de los escolares

En lo relativo a la utilidad y transferencia de la formación recibida, los monitores señalan que ésta es útil y enriquecedora para su desarrollo personal y profesional, ofreciéndoles la oportunidad de trabajar contenidos deportivos con escolares y aumentar así su experiencia y bagaje docente, tal como se presenta en las Figura 5. 
[...] me parece muy interesante para el alumnado que este cursando los grados de Primaria o Infantil, ya que nos aporta experiencia para un futuro, ya sea para las prácticas o para cuando ejerzamos de profesor. (ME03MOBE, p. 90)

Figura 5.

Fragmento de una memoria sobre la utilidad y transferencia de la formación recibida

\section{Conclusiones}

Tras haber presentado de forma abreviada los principales resultados de este estudio, llegamos a las siguientes conclusiones: 1) los monitores están satisfechos con la formación recibida en el PIDEMSG, en la cual está implícita la evaluación formativa, permitiendo la mejora continua de la calidad del proceso de enseñanza-aprendizaje; aunque habría que conseguir una mayor implicación de los monitores en el proceso formativo, y 2) debido al proceso continuo de evaluación los monitores señalan que mejoran sus cualidades docentes (futuros maestros y maestros titulados), a través de la experiencia y trabajo con escolares, lo que permite ir mejorando el proceso de enseñanza y aprendizaje que desarrollan para así favorecer aprendizajes significativos en los escolares.

\section{Referencias}

López-Pastor, V.M. (Coord.) (2006). La evaluación formativa y compartida en educación física. De la crítica al modelo tradicional a la generación de un sistema alternativo. Revisión de 12 años de experiencia. Efdeportes.com, 94. Recuperado de https://www.efdeportes.com/efd94/eval.htm

Martínez-Scott, S., López-Pastor, V.M. \& Pérez-Brunicardi, D. (2012). Diseño y desarrollo de un programa integral de deporte escolar municipal. Revista Pedagógica de Educación Física ADAL, 7-13.

Pérez-Brunicardi, D., Álvaro-Garzón, M., \& López-Pastor, V.M. (2018). (Coords.). El deporte escolar en el municipio de Segovia. Veinte años de innovación. Segovia: Universidad de Valladolid. 